Original Research Paper

\title{
An Ontological Approach for Dental Extraction Decision Making and Knowledge Dissemination - A Pilot Study for Dental Extraction Forceps
}

\author{
Shanmuganathan Vasanthapriyan and Kuhaneswaran Banujan \\ Department of Computing and Information Systems, Sabaragamuwa, \\ University of Sri Lanka, Belihuloya, Sri Lanka
}

Article history

Received: 04-04-2019

Revised: $27-05-2019$

Accepted: 22-06-2019

Corresponding Author:

Shanmuganathan

Vasanthapriyan

Department of Computing and Information Systems,

Sabaragamuwa University of

Sri Lanka, Belihuloya, Sri

Lanka

Email: priyan@appsc.sab.ac.lk

\begin{abstract}
Tooth extraction is one of the most usual surgical procedure in the field of dental. Not having proper knowledge of tooth and extraction instruments may cause too much complexity in extraction procedure or even some damages to patients' jaws. Mainly when using extraction forceps, the proper forceps should be used according to the teeth and the situation. So, it is very much important to have a sound knowledge of the instruments to be used, especially on extraction forceps. So, the knowledge of extraction forceps should be disseminated properly. After identifying this need, as a first stage, we gathered the information regarding the dental extraction forceps from the experts in the field. Then we started developing ontology as a second stage. Finally, the Knowledge Management (KM) Portal, which helps to share the knowledge of dental extraction, was developed. Since the quality and the accuracy of the ontology is the key in this research, it was evaluated and validated by using inbuilt $\mathrm{FaCT}++1.6 .5$ reasoner, online validator OOPS! and ontology experts as an iterative approach. It was also evaluated by using ontology non-experts in the final stage as an application-based (field test) evaluation. A questionnaire survey was conducted to the users to evaluate the KM portal (i.e., ontology). The results show that $85 \%$ of them are agreed and strongly agreed on the usefulness of the system. We confidently believe that our novel approach on dental extraction forceps KM portal can support all the dental related personnel to improve the knowledge and helpful in learning practices. Our next step is to model the ontology for the whole extraction process.
\end{abstract}

Keywords: Dental Extraction Forceps, Ontology, Knowledge Dissemination, KM Portal

\section{Introduction}

Knowledge means facts, information and skills developed through experience or theoretical and practical education. Knowledge sharing is an activity through which knowledge acquired is exchanged among people, communities, or organizations. In order to share knowledge, it should be structured and complete. But in the real world, in some certain cases, varied terminologies are used to express the same concept. The knowledge is not reaching everybody because of this unstructured, incomplete, general nature and varied formats of the information (Walisadeera et al., 2015). Computers should have to understand the meaning of the information distinctly in order to response for answering intelligently. Then only we can disseminate knowledge without any problem. Semantic web enables this understanding to computers (Choksi and Jinwala, 2015).

Further, ontologies are the most powerful tool for representing knowledge presented in the semantic web (Vasanthapriyan et al., 2017b). It is one of the most suitable knowledge management tools for supporting the representation of knowledge and processing, storing and retrieving knowledge. Ontologies built in a given domain must be foundational and strong enough to withstand with regard to representation of the meaning, to stop human dependent semantic bias (Park et al., 2007). Ontology models the concepts that reflect the reality of the world. It also defines common terms in a domain of interest (Trappey et al., 2013). 
Ontologies are widely used for various purposes such as Natural Language Processing (NLP), Knowledge Management (KM), e-commerce, intelligent integration of information and in the semantic web (Walisadeera et al., 2013a). A structured view of domain knowledge can be represented by an ontology. It also acts as a storage of concepts in the specific domain (Walisadeera et al., 2013b; Vasanthapriyan et al., 2017b). This structured view is essential in order to facilitate knowledge sharing and aggregation, information retrieval and question answering. Ontology provides precise and well-defined relationships, inference mechanism, strong semantic capabilities and reasoning support. Therefore, ontology can be applied in order to find the responses to queries within a specified context (Walisadeera et al., 2013b; Vasanthapriyan et al., 2017b; 2017a) in the domain of dental extraction. According to Gruber (1995), "an ontology is an explicit specification of a conceptualization".

A tooth is one of the hardest parts in most of the vertebrates. It is a calcified structure and situated inside jaws (Wikipedia, 30 November 2017). Tooth performs a very important function in the human body. It helps in breaking down of foods which aids the digestion easy. It also gives aesthetic value to the appearance of a person. Dentistry is a branch of medicine which deals with the dentition, related structures and tissues in the face. Removal of teeth from its socket in the jaws is known as a dental extraction (Kolosovas-Machuca et al., 2016; Wikipedia, 30 November 2017). There are many reasons for dental extractions but mainly it is done if a tooth has been damaged by decay or broken (Anyanechi and Chukwuneke, 2012). Other reasons are such as a crowded mouth, infection, supernumerary or malformed tooth and even because of cosmetic purposes (to remove tooth of poor appearance). Oral surgery is the branch of dentistry that deals mainly with extractions (Wikipedia, 30 November 2017). There are two types of extractions performed in the dental field:

- A simple extraction, which is performed on a tooth which is visible inside the mouth. In a simple extraction procedure, the dentist will hold the tooth with specialized pliers called "extraction forceps" and move them front and back in order to loosen the tooth from the jaw before getting rid off the tooth (Cclyer, 1986, Wikipedia, 30 November 2017)

- A surgical extraction, which is a more complex like other surgical procedure
The dental extraction forceps is an exaggerated version pair of pliers (Cclyer, 1986) which is specially used for dental extraction purposes. It is made up of three parts; the blades, the joint and the handles (Heimann, 1977). In general, the blades and handles are in the same line for forceps which are designed for the extraction of maxilla (upper) anterior (front) teeth as shown in Fig. 1a. For the maxilla posterior (back) teeth the handles form a curve with the blades as shown in Fig. 1b. In forceps used for the extraction of mandibular (lower) teeth, the blades and handles are at an angle of approximately ninety degrees between them as shown in Fig. 1c (Cclyer, 1986). When we take a simple extraction process, dental extraction forceps plays an important role (Cclyer, 1986) as it is the key to pull the tooth.

\section{Objectives}

The main objective of this work is to contribute to an improvement in the management and usage of dental extraction forceps in hospitals and as well as to create a base for a web portal to disseminate the dental extraction forceps knowledge for students by developing an ontology-driven solution which organizes and describes clearly related knowledge. This would assist the doctors and their assistants in the dental hospitals to share dental extraction forceps new experiences and it will be converted to new knowledge as well.

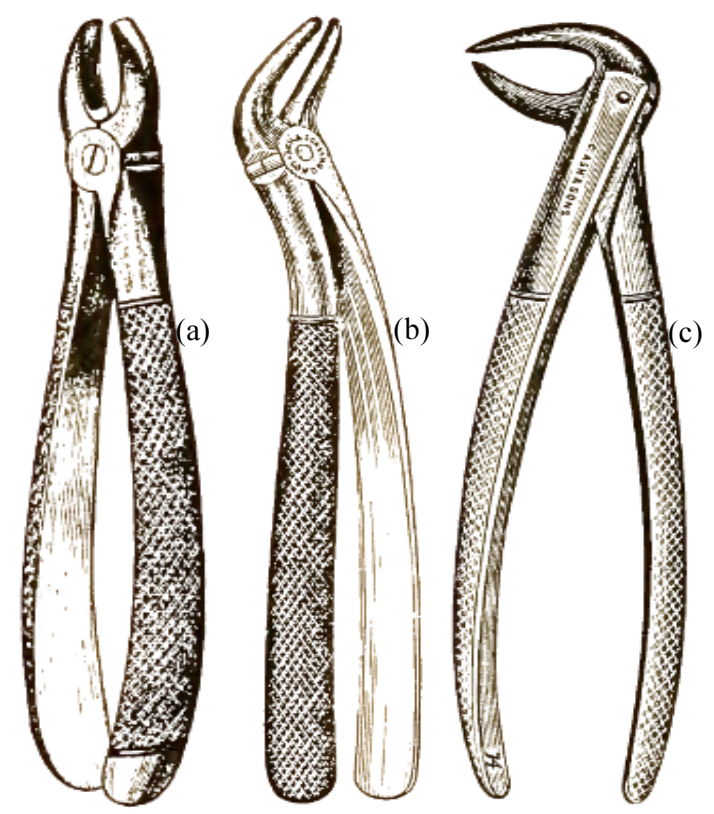

Fig. 1: (a) Forceps design for maxilla anterior teeth (b) Forceps design for maxilla posterior teeth (c) Forceps design for mandibular teeth 
This research was initiated (Vasanthapriyan, 2018) and the extension of the work is presented in this paper. The remainder of the paper is organized as follows. Section 2 describes the literature review and the related work. Section 3 explains the need for a new dental extraction forceps ontology Section 4 discusses our design methodology and section 5 concludes this paper with discussions and presents directions for future work.

\section{Literature Review and Related Works}

Using ontologies for modelling knowledge in the health sector is an active research field (Garcia-Valverde et al., 2014). An ontology provides a way to compile medical knowledge in a reusable, shareable, machine-understandable way (Park et al., 2007). Even though health sector is being supported by number of biomedical ontologies such as GALEN, the Unified Medical Language Source, the Systemic Nomenclature of Medicine which focus on general scope of the biomedical domain (Kuziemsky and Lau, 2010) and the Gene Ontology (GO) which is one of the earliest and most frequently used vocabularies ( $\mathrm{Hu}$ et al., 2016), there are a very few ontology on dental domain on the health sector. Since the ontology-based systems handle reusable terminology resources, they can be used to improve the management of complex systems for various contextual information (Garcia-Valverde et al., 2014).

The information needed for a domain may be available from books, leaflets, notices, online resources, mass media, etc. The information is also in various formats such as text, image, audio, video, etc. (Walisadeera et al., 2013b). The use of ontologies in the health domain mainly focused on the representation and re-organization of medical terminologies. Ontologies can help build more powerful and more interoperable information systems in healthcare. The most significant benefit that ontologies in the health sector are its ability to support the integration of knowledge and data (Pisanelli, 2004). Even though ontologies are used in the Information System (IS) design, the ontology development in the health sector is more challenging because of its complexity and the level of detail in it (Kuziemsky and Lau, 2010).

Having discovered this research gap, we have focused on our attention to developing a dental extraction forceps ontology to represent information needs according to tooth extraction context. That is, we intend to develop an ontology-based knowledge framework to manage extraction forceps-related knowledge.

\section{Need for Dental Extraction Forceps Ontology}

There are 20 teeth on a child called as primary dentition and 32 teeth on an adult called as permanent dentition. Further, each tooth has a crown and a root. Generally, when extraction is done, the root also comes with the crown. But in some special cases like the broken tooth, while extracting crown, roots stay in the jaw socket (Cclyer, 1986). Further, some teeth are very difficult to extract from its socket for various reasons. Some of them include tooth's position, grip power of the jaw to the tooth, the shape of the tooth roots, the strength of the tooth and the unity of the tooth.

All the tooth in human is not in the same shape and size. Since various types of teeth perform various functions, their shape and size vary. Jaws are short, long, straight, curved on the flat and angled on the flat or inline. So, each tooth needs to be extracted using different types of dental extraction forceps. So, dental forceps are shaped to accommodate different teeth without splintering them whilst powerful leverage is applied via the handles in order to loosen roots prior to extraction.

During the extraction process, the force to be applied should strictly be limited to the tooth that is to be extracted. Many cases of simple extraction procedures, before proceeding the extraction process, the forces are diverted from the tooth to areas such as bone surrounding the tooth. Further, if the tooth break on extraction, the removal of its root becomes very much more difficult (Heimann, 1977). In the worst case, this may cause surgery and it is very painful.

Choosing the appropriate extraction forceps is the important part for the protection of jaws and another neighboring tooth which will be affected if the forceps slips away while extracting. If the specific extraction forceps are not used for a tooth, then there will be more complications (OLurel et al., 2014) such as incomplete extraction in which a tooth root remains in the jaw, prolonged bleeding, swelling, bruising, nerve injury or even extraction of the wrong tooth (Wikipedia, 30 November 2017).

If proper extraction forceps are used by the dentist, his or her extraction will be easy as each extraction forceps are made by using the knowledge of physics. For example, extraction forceps are made smaller in size for children in order to apply less force and larger in size for adults in order to apply more force. If we take another example, the tooth on deep end in the jaw (for example wisdom tooth) needs a different mechanism to extract than the tooth in the front part of the jaw (for example central incisor) (Cclyer, 1986). So, extraction forceps are made "L" shape for the deeper end and straight for central area. Therefore, usage of specific extraction forceps for the specific tooth is very important for patients' health as well as for the easiness of the dentist. Moreover, when extracting maxillary teeth, the extraction forceps are held with palm under the handle (Fig. 2a). Forceps are held with palm on top of extraction forceps for extracting mandibular teeth (Fig. 2b). If it is not followed, there will be more complications as mentioned above. 


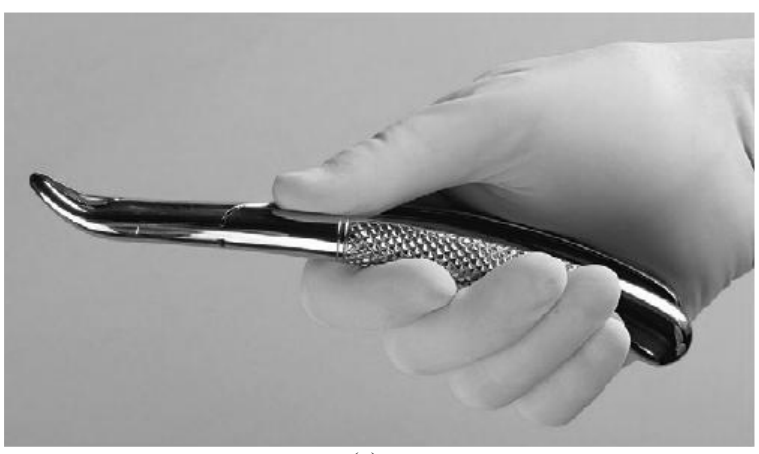

(a)

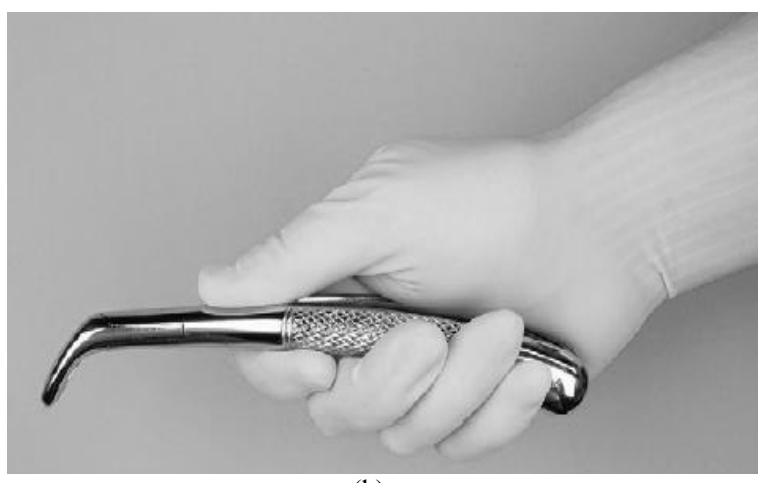

(b)

Fig. 2: Forceps holding method for (a) maxilla teeth (b) mandibular teeth

Further, different terminologies are used for the same thing. For example, some say "upper", some say "maxilla". So all those terminologies should be unified. The information and knowledge need to be provided in a structured and complete way and in a context specific manner. In our research, dental extraction forceps are the context.

Although the current ontologies are designed to minimize ambiguities and redundancy, none of these ontologies have been used in dentistry (Park et al., 2007). By considering the importance of sharing the knowledge on dental extraction, as a first move, the ontology for dental extraction forceps is developed. The goal of our work is to use the ontology to create a knowledge base as input for a software program that improves the capturing of information and the creation of knowledge (Trappey et al., 2013).

\section{Research Design}

In this section, we present our design approach used in our research. We divide our approach into three main parts; (i) Development of the dental extraction forceps ontology, (ii) Development of the KM Portal for dental extraction forceps and (iii) Evaluation and validation which is done for the both developed ontology and as well as for KM portal. Our overall methodological framework is shown in Fig. 3.

\section{Contextual Information}

According to Dey (Abowd et al., 1999), context is any information which can be used to describe the situation of a participant in a meaningful way. Our main target groups are dentists and people associated with dental in Sri Lanka such as assistants in dental hospitals, lecturers, students, researchers and information specialists. In order to identify dental extraction context clearly, we have extracted domain specific knowledge by using the following reliable knowledge sources:

- Related field experts from Sri Lankan universities, Sri Lankan hospitals by using formal and informal interviews, discussions

- Research articles, online articles and books (Wikipedia, 2017; Park and Kim, 2006; Malden, 2001; Kolosovas-Machuca et al., 2016; Cclyer, 1986; Heimann, 1977; Balaji, 2013; Anyanechi and Chukwuneke, 2012)

- Newspapers, radio and television

\section{Data Collection}

Grounded theory was used for the data collection (Strauss and Corbin, 1994). An extensive literature survey and expert collaboration were used to get relevant data. Two dentists with extensive knowledge of dental (mainly on extraction) and an expert on ontology engineering took part. Dental related personnel from various hospitals and dental lecturers (three) were also interviewed regarding the idea of unifying forceps catalogs and the information that should be included in it.

There are three international standard systems for naming teeth: (i) the universal numbering system, (ii) the Palmer notation method and (iii) the two-digit FDI world dental federation notation. In this paper, we followed two-digit FDI world dental federation notation WHO and by other organizations such as the International Association for Dental Research. It provides a system for designating teeth or areas of the oral cavity using two digits (Park and Kim, 2006). The high-level class hierarchy is shown in Fig. 4.

\section{Competency Questions (CQs)}

Competency Questions (CQs) are a set of questions that the ontology must be capable of answering using its axioms (Grüninger and Fox, 1995). We can verify our ontology by checking whether our ontology contains all the necessary and sufficient axioms that correctly answer the CQs (Vasanthapriyan et al., 2017 b). Our ontology aims to answer the following CQs shown in Table 1. 


\section{Ontology Modelling}

Modelling ontology is a time-taking process (Walisadeera et al., 2013b) and hence requires appropriate tools (Ayalew et al., 2018). Further, constructing a domain ontology from the beginning is a complicated task. After reviewing all the methodologies in literature, we selected Grüninger and Fox's methodology (Grüninger and Fox, 1995) for our modelling of dental extraction forceps ontology. The reason for selecting Grüninger and Fox's methodology is, it publishes a formal approach for designing the ontology and also it provides a framework for evaluating the developed ontology (Vasanthapriyan et al., 2017b). It focuses on building ontology based on first-Order Logic (FOL) by providing strong semantics. We used OWL2 Web Ontology Language (Consortium, 2012) for modelling ontology, i.e., for formalizing relationships between concepts. We choose OWL because it is a markup language with a formal, logical semantics (Schuurman and Leszczynski, 2008).

Prior to answering the CQs, we need to identify basic ontology components such as main concepts (e.g., Dentition, Forceps, Positions, etc.), their properties (e.g., Forceps has properties: holding position, shape, etc.) and relationships between concepts (e.g., Forceps hasUsedToExtract Dentition, Dentition hasAPosition Position, etc.). We also define the subclasses by considering instances of the concepts and their properties. For example, Tooth Specific Name has Canine, Incisor, Pre-molar and Molar as its subclasses. These subclasses are related to their superclass by "is a" relation (Vasanthapriyan et al., 2017b). All these things were made from the data collected.

The associative relationships are to identify the concepts and relationships with meaningful relations and to define the relationships and their inverse relationships (if exists). For example, there is an associative relationship with the inverse relationship between Dentition and Position: Dentition hasAPosition Position, Position isAPositionOf Dentition. Table 2 shows some associative relationships including their inverse used in the ontology modelling.

Datatype properties link an individual to an XML Schema Datatype value or an RDF literal. In other words, they describe relationships between an individual and data values. A datatype property can also be used in a restriction to relate individuals to members of a given datatype. Some of the data properties used are hasShape, hasHandleSize, hasJointBeaks, hasMovement, hasPosition.

The ontology was implemented by using the Protégé-OWL Ontology Editor 5.1. Part of the dental extraction forceps ontology is shown in Fig. 5.

We used Description Logic (DL) for our work because we used OWL 2 Web Ontology Language for modelling the ontology. DL is a Decidable Fragment of FOL (Walisadeera et al., 2013a) and is more expressive than primitive FOL. CQs were evaluated using the DL expressions. The DL query facility available in the ProtégéOWL Ontology Editor 5.1 was used for this purpose. Some of the DL query and their answers are shown in Table 3.

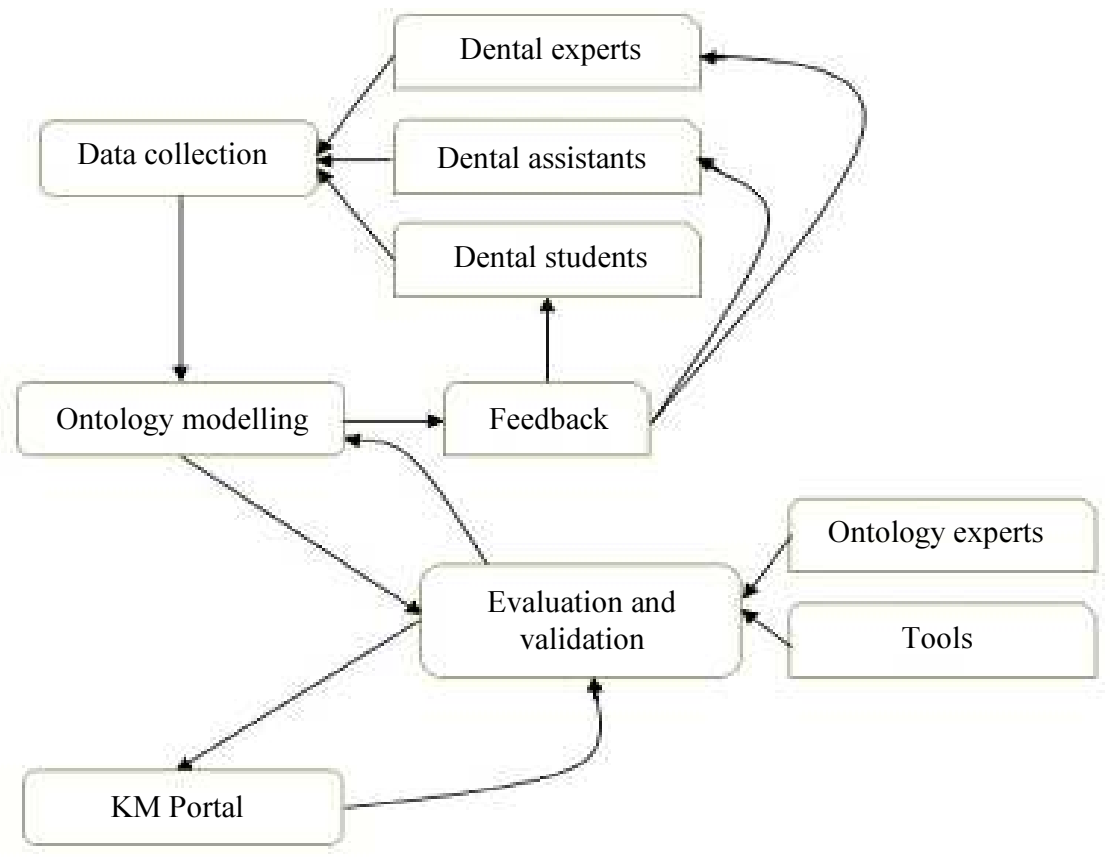

Fig. 3: The overall methodological framework for the development of the KM portal for dental extraction forceps 


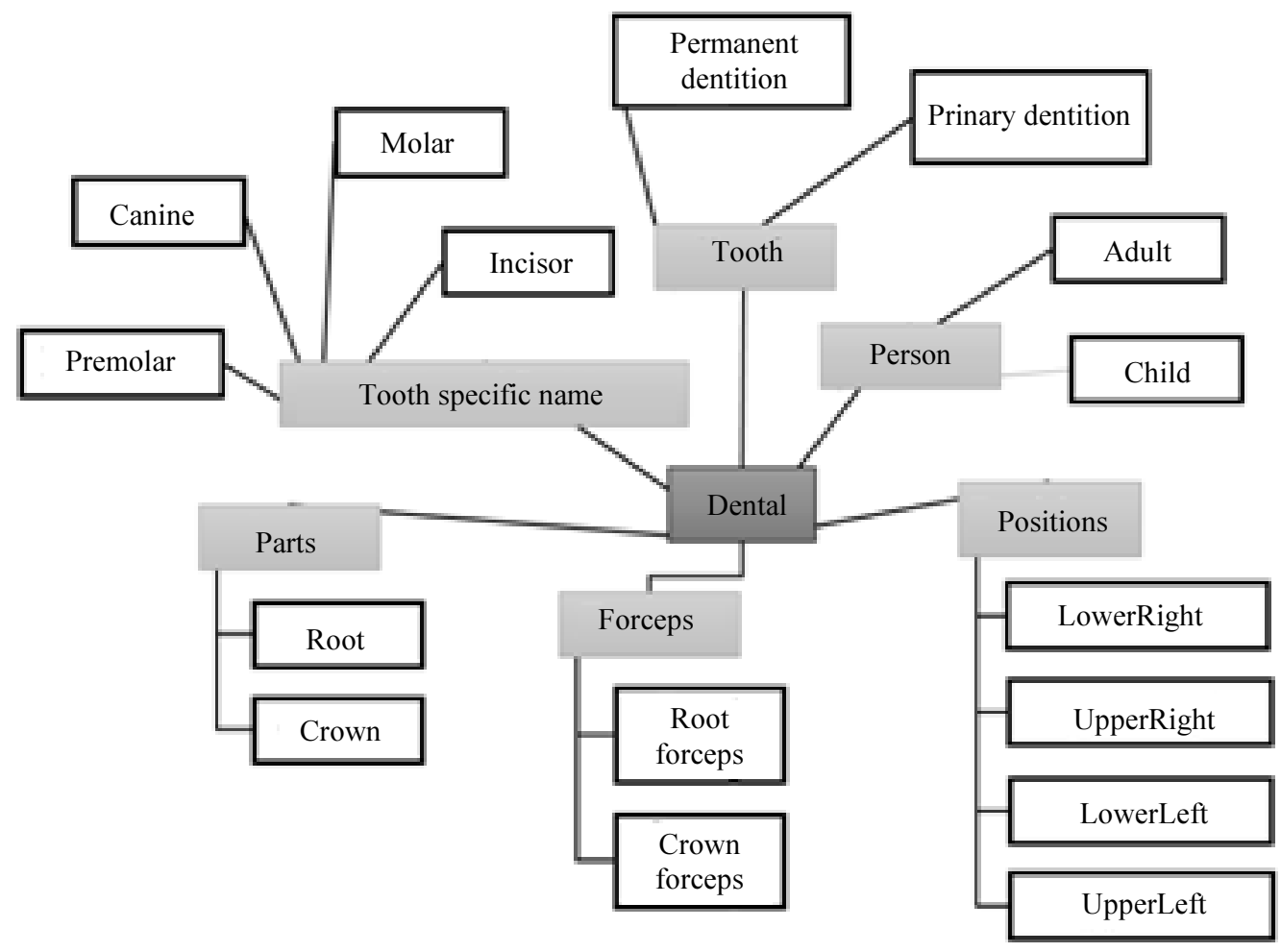

Fig 4: The high-level class hierarchy of dental extraction forceps ontology

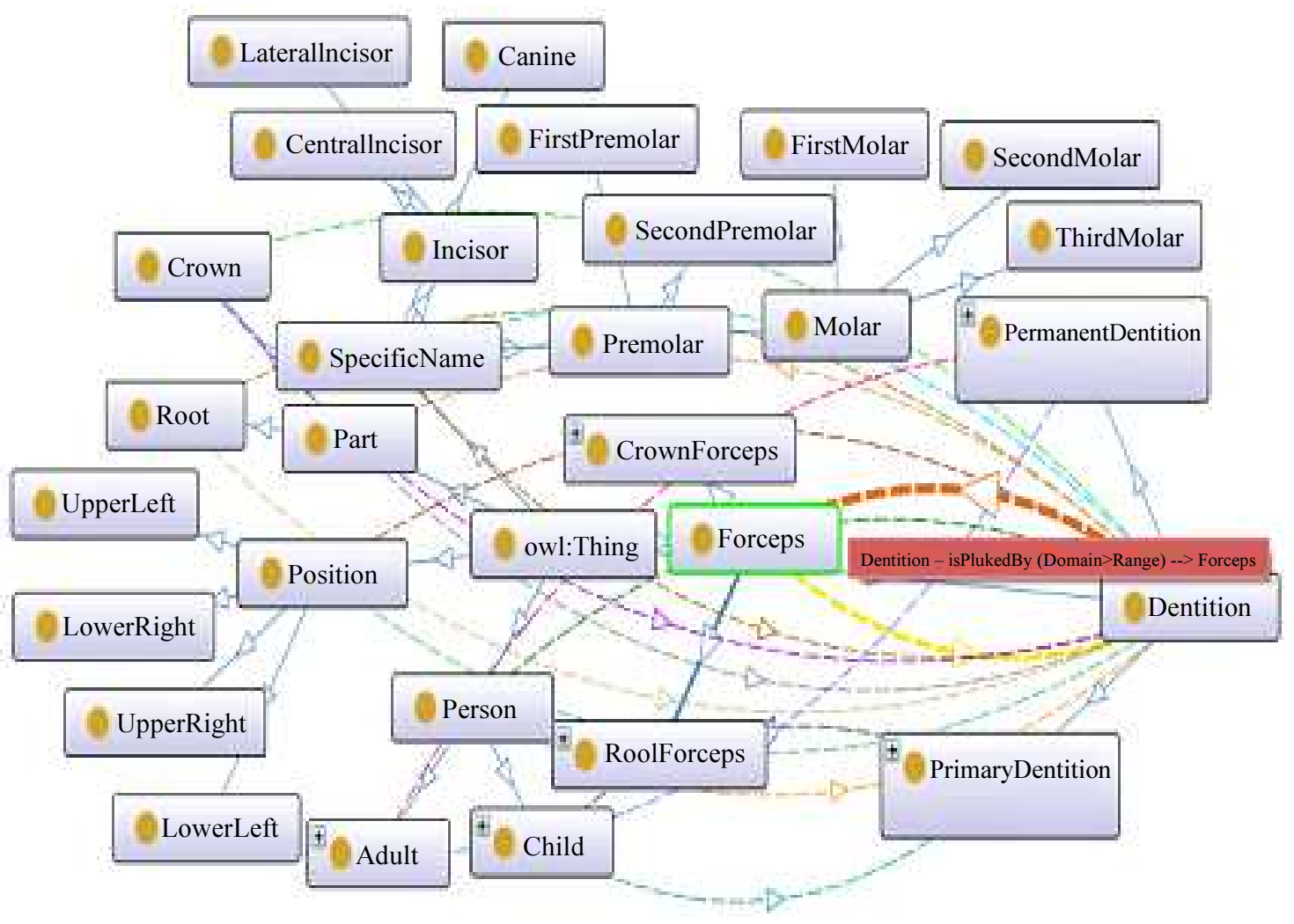

Fig. 5: Part of the dental extraction forceps ontology 
Table 1: Competency questions

Competency questions

What is the position of canine in the lower right side of a child in the mouth?

Which extraction forceps is used to extract adult third molar in the upper left side?

Which tooth is in the lower right side of an adult?

Which teeth are in the upper left side of an adult?

Which extraction forceps are L-shaped?

What are the dentitions consists of specific name canine?

What type of movement is applied to the third molar of the upper left side of an adult

How to hold the dental extraction forceps in order to extract upper teeth?

What are the main parts associated to tooth regarding extraction?

What is the position of central incisor of a child in the mouth?

What is the tooth number (dentition) of lower left lateral incisor of an adult?

What is the tooth of lower left central incisor of a child in the mouth?

Which extraction forceps are needed to extract the lower right side second molar of a child?

Which extraction forceps are needed to extract the root of left second premolar of an adult?

Which extraction forceps are S-shaped?

Which extraction forceps is used to extract crown of the adult third molar in upper left side?

What type of dentition is for a child?

Table 2: Associative relationships including their inverse

\begin{tabular}{lll}
\hline Concept & Relationship & Concept \\
\hline Dentition & has a Specific Name, is a specificname of & Specific Name \\
Forceps & has Used to Extract, is Extracteded by & Dentition \\
Dentition & has a Position, is a Position of & Position \\
Adult & has Permanent Dentition, is Permanent Dentition of & Permanent Dentition \\
Dentition & has Part Root, ispartroot of & Root \\
\hline
\end{tabular}

Table 3: CQs, DL query and their answers

\begin{tabular}{|c|c|c|}
\hline Competency questions & DL query & Answers \\
\hline What forceps are used to pluck T55 dentition? & Forceps and hasUsedToPluck value T55 & $\begin{array}{l}\text { Instances (2) Child } \\
\text { UpperMolarCF } \\
\text { ChildUpperRF }\end{array}$ \\
\hline What is the position of $\mathrm{T} 34$ dentition? & Position and isPositionOf value T34 & Instances (1) LowerLeft \\
\hline What is the specific name of T28 dentition? & SpecificName and isSpecificNameOf value T28 & Instances (1) ThirdMolar \\
\hline Which extraction forceps are in "L" shape? & Forceps and hasShape value "L" & $\frac{\text { Instances (2) }}{\text { BayonetRF }}$ BayonetCF \\
\hline Which extraction forceps have joint beaks? & Forceps and hasJointBeaks value "Yes" & $\begin{array}{l}\text { Instances (9) BayonetCF } \\
\text { ChildLowerMolarCF } \\
\text { ChildUpperMolarCF } \\
\text { LowerMolarCF } \\
\text { LowerPremolarCF } \\
\text { LowerWisdomCF } \\
\text { UpperLeftMolarCF } \\
\text { UpperPremolarCF } \\
\text { UpperRightMolarCF }\end{array}$ \\
\hline
\end{tabular}

\section{Development of the KM Portal}

Web resources are provided with machine-readable descriptions of data, programs and infrastructure in the semantic web. It is an extension of the current WWW. Semantic webs are better enabling in computers and people to work in co-operation. According to this viewpoint, more attention has been given to semantic web technologies and ontologies (Vasanthapriyan et al., 2017a). In this section, we describe the designing of knowledge framework to share dental extraction forceps knowledge. It was built upon the Java J2EE distributed component environment. The five layers of our knowledge framework are shown in Fig. 6 .

Ontology Layer: The dental extraction forceps ontology, which includes domain, rules, axioms, etc. is in the ontology layer. This layer is constructed on OWL DL. OWL DL includes a Terminology Box (TBox), an Assertion Box (ABox) and a rule base.

Experience Sharing and Knowledge Validation 
Layer: This layer comprised of two parts; (i) Experience sharing and (ii) Knowledge validation. Dental doctors can share their experience through this layer. For example, other standard extraction forceps used by them and which tooth is related to it and the properties related to them can be fed into the system. The semantic data generator is used to transform the shared knowledge into semantic data.

Storage Layer: Triple-store, which stores RDF triples, was used in this layer. Using SPARQL, the queries were made. Since Jena TDB is a component of Jena for RDF storage and query, it was selected in this study. It supports the full range of Jena APIs and can be used as a high performance of the RDF store on a single machine.

Reasoning Layer: OWL instances were reasoned in terms of OWL classes and properties in Semantic Web Rule Language (SWRL) rules. Significantly, more complex relationships and restrictions between concepts were expressed in such rules. Protégé SWRL Editor Plugin tool available in Protégé-OWL Ontology Editor 5.1 was used to generate dental extraction forceps rules. It was supported by the Jess Rule Engine.

Dental Extraction Forceps Knowledge Sharing Layer: Dental extraction forceps knowledge sharing layer was created in order to show how our dental extraction forceps ontology could be used to share knowledge collected from dental experts. Knowledge sharing layer has two main functionalities, which use semantic web technologies; (i) Basic search and (ii) Advanced search. In order to retrieve dental extraction forceps knowledge from the semantic data storage, SPARQL has been used as the query language.

\section{Evaluation and Validation}

In order to avoid the defects when using the ontology, its quality should be verified and validated (Poveda-Villalón et al., 2012). Therefore, the last stage of our methodology consisted of an evaluation of the ontology by experts in the field ontology, medical experts (dental related personnel) and by using inbuilt tools. We did the evaluation and validation throughout the whole process.

\section{Internal Evaluation and Validation}

Reasoner is a piece of software which has an ability to infer logical consequences from a set of asserted facts or axioms. We used inbuilt $\mathrm{FaCT}++$ 1.6.5 reasoner of Protégé-OWL Ontology Editor 5.1.

Further, in order to detect potential pitfalls which can lead to modelling errors, we used an online ontology evaluator called OOPS! (http://oops.linkeddata.es/) (Poveda-Villalón et al., 2012). This evaluator evaluates human understanding, logical consistency, modelling issues, real-world representation and semantic applications for the developed ontology. The summary of the pitfall encountered, brief description and description of how those are handled are shown in Table 4.

\section{External Evaluation}

\section{Expert Evaluation}

Medical experts will face huge difficulties when building the ontologies. Especially in the use of existential $(\exists)$ and universal $(\forall)$ quantifiers. In addition, we cannot expect the assistance from dental experts in extending the ontology axioms. Because of this, the modification of the correctness of the ontology is done by knowledge engineers (Maragoudakis and Maglogiannis, 2011). Therefore, we evaluated the ontology with the help of two ontology expert by examining the deficiencies of the artifacts we used. There were many methods to evaluate the ontologies in the literature (Vasanthapriyan et al., 2017b). Our ontology experts considered the following layers to perform the evaluation; (i) syntax, (ii) vocabulary, (iii) structure, (iv) semantics and (v) context and (vi) representation. This methodology allows us a better focus because each level has a different evaluation objective. Table 5 shows some of the suggestions and improvements highlighted by the ontology expert.

\section{Non-Expert Evaluation and Field Test}

The quality and the level of user satisfaction with the knowledge provided by the ontology are evaluated in this stage. To carry out our non-expert evaluation and field test from dental experts and dental related personnel, the developed KM Portal was hosted locally in one of the private hospitals in Sri Lanka. The evaluation study comprised of a training session in which dental experts and dental related personnel were given a brief introduction to the project and what is expected from them. It is followed by a demonstration of how to use the system. A separate questionnaire was designed in English with the fivepoint Likert-type scale to capture respondents' selfreported attitudes where respondents had to make their level of agreement on of the followings:

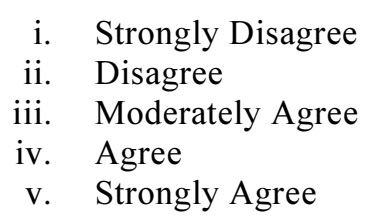

Scores 1, 2, 3, 4 and 5 were assigned respectively for the above-mentioned categories. The profiles and demographics of the participants (Employed Group, work experience, job description and qualification) were questioned as a first part and continued with questions focused to check whether developed 
ontology was able to (i) express dental extraction forceps knowledge (ii) support dental extraction forceps knowledge sharing (iii) support dental extraction forceps knowledge retrieval and (iv) user satisfaction. We limited the time period to fifteen days to collect the questionnaire data. This was performed by three dental experts who are specialized in dental extraction, five assistants in the dental field, two dental lecturers and five dental students. The dental experts and dental lecturers were given a separate questionnaire to check the quality of the system along with the questionnaire, which was given to the rest of the evaluation group.
When the dental experts and the dental lecturers were asked to assess the content of the KM Portal, four of them agreed (Likert Scale - 4) that correct contents have been included. All of the dental experts and the dental lecturers participated believed that the standards have been maintained (Likert Scale -4 and 5). Table 6 shows the summary of the outcomes for some of the questions in the special questionnaire of the dental experts and the dental lecturers.

The ontology was redesigned based on the feedback from the field test. Evaluators' requirements and suggestions were also gathered for future refinements of the ontology.

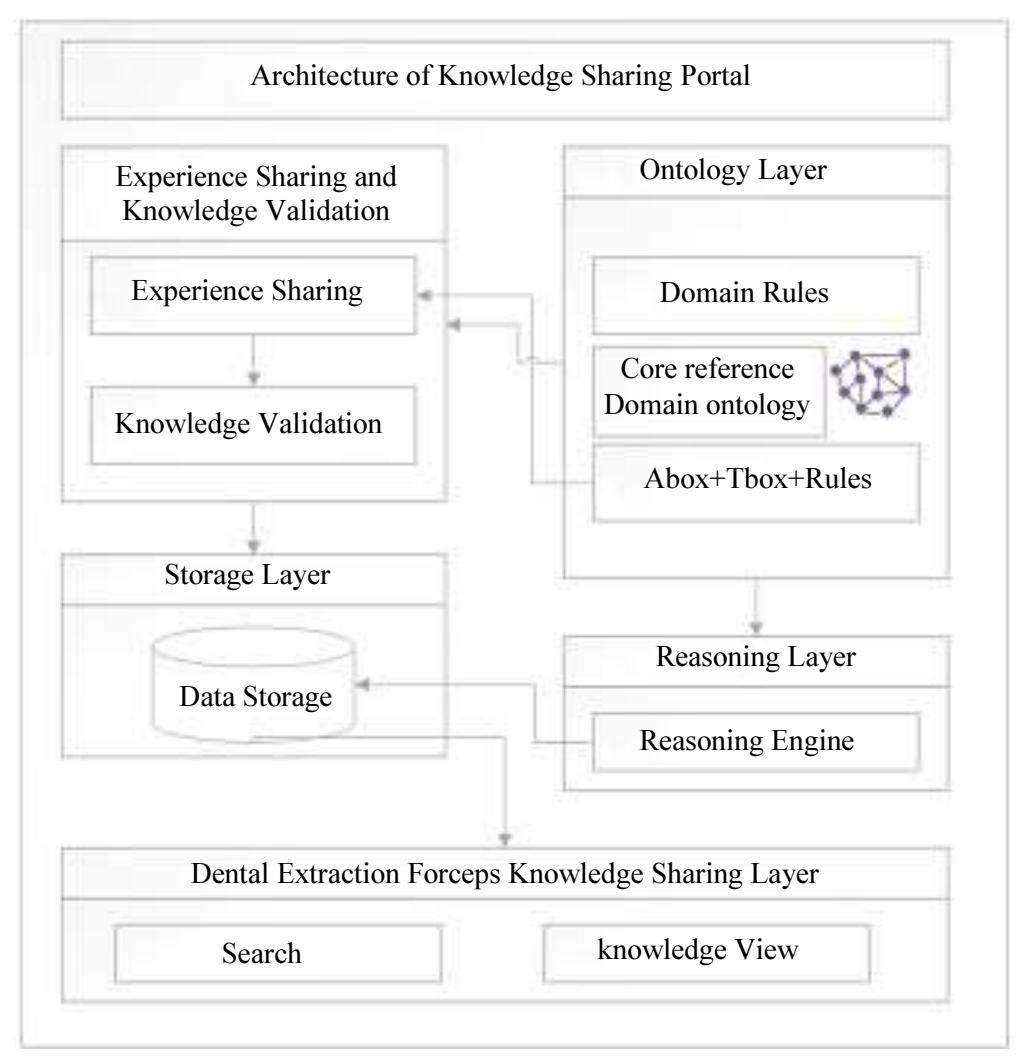

Fig. 6: The architecture of KM portal

Table 4: Pitfall, description and solution proposed

\begin{tabular}{|c|c|c|}
\hline Pitfall & Description & Solution \\
\hline $\begin{array}{l}\text { Inverse relationships not explicitly } \\
\text { declared ( } 24 \text { cases } \mid \text { Minor })\end{array}$ & $\begin{array}{l}\text { Except for the symmetric properties, others } \\
\text { do not have an inverse relationship. }\end{array}$ & Included missing inverse relationships \\
\hline Missing annotations (296 cases | Minor) & $\begin{array}{l}\text { Not providing understandable } \\
\text { annotations to ontology elements. }\end{array}$ & Included the ontology annotations \\
\hline $\begin{array}{l}\text { Using different naming conventions in } \\
\text { the ontology ( } 5 \text { cases } \mid \text { Minor })\end{array}$ & $\begin{array}{l}\text { Ontology elements are not named } \\
\text { in the same convention }\end{array}$ & $\begin{array}{l}\text { Corrected by using the uniform } \\
\text { naming convention }\end{array}$ \\
\hline $\begin{array}{l}\text { Creating unconnected ontology } \\
\text { elements ( } 3 \text { cases | Minor) }\end{array}$ & $\begin{array}{l}\text { Ontology elements are created, with no } \\
\text { relation to the rest of the ontology }\end{array}$ & Relationships are created \\
\hline $\begin{array}{l}\text { Missing domain or range in properties } \\
\text { (6 cases | Important) }\end{array}$ & $\begin{array}{l}\text { Object and (or) datatype properties } \\
\text { without domain or range }\end{array}$ & Added the missing domain and range \\
\hline $\begin{array}{l}\text { Defining wrong inverse relatio } \\
\text { ships ( } 2 \text { cases } \mid \text { Critical })\end{array}$ & $\begin{array}{l}\text { Two relationships are defined as } \\
\text { inverse relations when they are not } \\
\text { necessarily inverse }\end{array}$ & Removed those relationships \\
\hline
\end{tabular}


Table 5: Review summary of ontology expert

\begin{tabular}{|c|c|c|}
\hline Discussion topic & Onto expert 1 & Onto expert 2 \\
\hline Standard syntax is used or not & Manchester syntax was followed & Manchester syntax was used \\
\hline Whether URIs are used for naming the nodes & All the nodes contained URIs & Each node was defined with URIs \\
\hline $\begin{array}{l}\text { Whether it describes } i s-a \text { relationship } \\
\text { between concepts }\end{array}$ & $\begin{array}{l}\text { All the classes followed the } \\
i s-a \text { relationship }\end{array}$ & Is- $a$ relationship exists for all classes \\
\hline $\begin{array}{l}\text { Tools used for checking the structure } \\
\text { of the ontology }\end{array}$ & Onto Graf was used to viewing the ontology & $\begin{array}{l}\text { Hierarchy, property relationships and levels } \\
\text { of details were checked using Onto Graf }\end{array}$ \\
\hline Availability of well and properly defined CQs & $\begin{array}{l}\text { CQs clearly explains the knowledge } \\
\text { of dental extraction forceps }\end{array}$ & $\begin{array}{l}\text { A very few CQs were needed to be } \\
\text { modified as highlighted }\end{array}$ \\
\hline The use of query languages for answering the CQs & DL query language was used & CQs were answered using DL queries \\
\hline Proper taxonomy followed or not & Onto Graf shows clear taxonomy & $\begin{array}{l}\text { Good view of the taxonomy is } \\
\text { viewed using Onto Graf }\end{array}$ \\
\hline
\end{tabular}

Table 6: Summary of the results for the outcomes for the special questions

\begin{tabular}{llll}
\hline$\#$ & Question & Mode (Likert scale) & Conclusion \\
\hline 1 & $\begin{array}{l}\text { Is all information regarding the dentalextraction forceps provided? } \\
\text { Is the information is sufficient to make a decision on which extraction } \\
\text { forceps to use for which tooth? }\end{array}$ & 4 & Agree \\
& $\begin{array}{l}\text { Can the system be used by the persons who } \\
\text { are having no knowledge in the dental field? }\end{array}$ & 5 & Very Easy \\
$4 \quad$ Whether the standard is maintained or not & 5 & Strongly Agree \\
\hline
\end{tabular}

\section{Discussions and Conclusion}

Dental extraction is an experience-based and knowledge-intensive activity, which mainly depends on the experience and the knowledge of the dental doctors and their assistants as well. KM in dental extraction aims to create an environment for continuous knowledge creation and knowledge sharing among dental doctors, dental assistants, dental lecturers, dental students and other researchers. Therefore, in this research, greater importance is given to knowledge for dental extraction and the potential benefits of managing dental extraction forceps knowledge. An ontological approach to represent the necessary dental extraction knowledge within the dental related personnel was developed:

i. The validity determines the quality of the ontology. That is why we evaluated our ontology in many different ways in order to improve the quality of the ontology

ii. We used inbuilt $\mathrm{FaCT}++1.6 .5$ reasoner of Protégé-OWL Ontology Editor 5.1 to check the logical consistency

iii. In order to detect potential pitfalls which can lead to modelling errors, we used an online ontology evaluator called OOPS

iv. Ontology experts were also used for evaluation purposes. Experts' responses, comments and suggestions were taken into account and ontology was redeveloped

v. At last, application-based evaluation was conducted with the ontology nonexperts but with the experts in the dental domain
As a summary, in this article, we presented an approach for (i) dental extraction forceps ontology modelling in order to represent dental extraction forceps domain knowledge, which includes dental extraction forceps concepts, properties and their relationships and (ii) the development of the KM portal. We confidently believe that our novel approach in dental extraction forceps KM portal can support other hospitals education institutes to improve the sharing of knowledge and learning practices. As a future work, we are planning to expand our domain area for the whole extraction process. It will be more complex work because there are many types of equipment used and the process is more complicated when dealing with surgical extraction. We are expecting more experience sharing process will be there if we could able to complete the KM portal for the whole extraction process.

\section{Acknowledgment}

We pay our sincere gratitude to the academic staffs of the Faculty of Dental Sciences of University of Peradeniya. Further, we acknowledge Dr. P. Pathmaja(BDS) and the other experts who have directly and indirectly involved in order to complete the research work successfully.

\section{Author's Contributions}

Shanmuganathan Vasanthapriyan: Designing of experiment (70\%), Laboratory and experiment (70\%), Interpretation and data analysis (70\%), Writing up (70\%).

Kuhaneswaran Banujan: Designing of experiment $(30 \%)$, Laboratory and experiment $(30 \%)$, Interpretation and data analysis (30\%), Writing up (30\%). 


\section{Ethics}

The author testifies that this article is original and contains unpublished material. All ethical standards that ensure scholarly integrity have been followed.

\section{References}

Abowd, G., A. Dey, P. Brown, N. Davies and M. Smith et al., 1999. Towards a better understanding of context and context-awareness. Handheld Ubiquitous Comput., 4: 304-307.

DOI: 10.1007/3-540-48157-5 29

Anyanechi, C. and F. Chukwuneke, 2012. Survey of the reasons for dental extraction in eastern Nigeria. Anna. Med. Health Sci. Res., 2: 129-133. DOI: $10.4103 / 2141-9248.105659$

Ayalew, Y., B. Moeng and G. Mosweunyane, 2018. Experimental evaluation of ontology-based HIV/AIDS frequently asked question retrieval system. Health Inform. J., 1: 460-468. DOI: $10.1177 / 1460458218775147$

Balaji, S., 2013. Burried broken extraction instrument fragment. Anna. Maxillofacial Surgery, 3: 93-93. DOI: $10.4103 / 2231-0746.110076$

Cclyer, J.F., 1986. Extraction of the Teeth. 1st Edn., Claudius Ash and Sons, Limited, London.

Choksi, A.T. and D.C. Jinwala, 2015. A novel way to relate ontology classes. Sci. World J.

Consortium, W.W.W., 2012. OWL 2 web ontology language document overview.

Gruber, T.R., 1995. Toward principles for the design of ontologies used for knowledge sharing? Int. J. Human Comput. Stud., 43: 907-928. DOI: 10.1006/ijhc.1995.1081

Grüninger, M. and M.S. Fox, 1995. Methodology for the design and evaluation of ontologies.

Heimann, W., 1977. Extraction forceps for upper molars. Google Patents.

$\mathrm{Hu}, \mathrm{Y}$., W. Zhou, J. Ren, L. Dong and Y. Wang et al., 2016. Annotating the function of the human genome with gene ontology and disease ontology. Bio. Med. Res. Int.

Kolosovas-Machuca, E.S., J.F. Pozos-Guillen, A.J. Campos-Lara and M. Pierdant-Perez, 2016. Pain measurement through temperature changes in children undergoing dental extractions. Pain Res. Manage.

Kuziemsky, C.E. and F. Lau, 2010. A four stage approach for ontology-based health information system design. Artificial Intell. Med., 50: 133-148. DOI: $10.1016 /$ j.artmed.2010.04.012

Malden, N., 2001. Surgical forceps techniques. Dental Update, 28: 41-44.

DOI: $10.12968 /$ denu.2001.28.1.41
Maragoudakis, M. and S.I. Maglogianni, 2011. A medical ontology for intelligent web-based skin lesions image retrieval. Health Informatics J., 17: 140-157. DOI: $10.1177 / 1460458211405009$

Park, S. and H.G. Kim, 2006. Dental decision making on missing tooth represented in an ontology and rules. Semantic Web-ASWC, 6: 322-328. DOI: $10.1007 / 1183602532$

Park, S.G., H.G. Kim and M.K. Kim, 2007. Tooth positional ontology represented in owl. Proceedings of the 12th World Congress on Health (Medical) Informatics, Building Sustainable Health Systems, (SHS' 07), IOS Press, Australia, pp: 2288-2288.

Pisanelli, D.M.. 2004. Ontologies. http://www.openclinical.org/ontologies.html

Poveda-Villalón, M., M. Suárez-Figueroa and A. Gómez-Pérez, 2012. Validating ontologies with oops. Knowl. Eng. Knowl. Manage., 6: 267-281. DOI: $10.1007 / 978-3-642-33876-2 \_24$

Schuurman, N. and A. Leszczynski, 2008. A method to map heterogeneity between near but nonequivalent semantic attributes in multiple health data registries. Health Informatics J., 14: 39-57. DOI: $10.1177 / 1460458207086333$

Strauss, A. and J. Corbin, 1994. Grounded theory methodology. Handbook Qualitative Res., 17: 273-285. DOI: 10.4324/9780203409527.ch13

Garcia-Valverde, T., A. Muñoz, F. Arcas, A. BuenoCrespo and A.A. Caballero, 2014. Heart health risk assessment system: A nonintrusive proposal using ontologies and expert rules. Bio. Med. Res. Int., 4: 12-12. DOI: 10.1155/2014/959645

Olurel, T.Z.O., R. Olurel, N. Örnek, N.B. Gökçjnar and K. Örnek, 2014. Branch retinal artery occlusion following dental extraction. Case Reports in Ophthalmological Medicine.

Trappey, C.V., T.M. Wang, S. Hoang and A.J. Trappey, 2013. Constructing a dental implant ontology for domain specific clustering and life span analysis. Adv. Eng. Informatics, 27: 346-357. DOI: $10.1016 /$ j.aei.2013.04.003

Vasanthapriyan, S., 2018. An ontological approach for dental extraction decision making and knowledge dissemination. Proceedings of the 18th International Conference on Advances in ICT for Emerging Regions, Sept. 26-29, IEEE Xplore Press, Colombo, Sri Lanka, Sri Lanka, 1-1.

DOI: 10.1109/icter.2018.8615487

Vasanthapriyan, S., J. Tian and J. Xiang, 2017a. An ontology-based knowledge framework for software testing. Int. Symposium Knowl. Syst. Sci., 3: 212-226. DOI: 10.1007/978-981-10-6989-5_18 
Vasanthapriyan, S., J. Tian and J. Xiang, 2017b. An ontology-based knowledge management system for software testing. Proceedings of the 29th International Conference on Software Engineering and Knowledge Engineering (EKE' 17), pp: 522-525. DOI: $10.18293 /$ seke2017-020

Walisadeera, A.I., A. Ginige and G.N. Wikramanayake, 2015. User centered ontology for Sri Lankan farmers. Ecol. Informatics, 26: 140-150. DOI: 10.1016/j.ecoinf.2014.07.008

Walisadeera, A.I., G.N. Wikramanayake and A. Ginige, 2013a. Designing a farmer centred ontology for social life network. DATA, 2: 238-247.

DOI: $10.1016 /$ j.ecoinf.2014.07.008
Walisadeera, A.I., G.N. Wikramanayake and A. Ginige, 2013b. An ontological approach to meet information needs of farmers in Sri Lanka. Proceedings of the International Conference on Computational Science and Its Applications, Springer, pp: 228-240.

DOI: $10.1007 / 978-3-642-39637-3 \quad 19$

Wikipedia, 2017. Dental extraction. https://en.wikipedia.org/wiki/Dental_extraction

Wikipedia, 30 November 2017. Dental extraction Online.

https://en.wikipedia.org/wiki/Dental_extraction 